\title{
Estudio teórico de algunas propiedades termodinámicas de los ácidos grasos obtenidos como destilados en la desodorización de aceites comestibles
}

\author{
Por M. M. Prieto', J. C. Bada Gancedo², M. I. Lombardía Prendes ${ }^{2}$ y E. Graciani ${ }^{3}$. \\ 'Departamento de Energía, ETS de Ingenieros Industriales de Gijón. Universidad de Oviedo. Edificio de \\ Energía. Campus de Viesques. Ctra. Villaviciosa, s/n. 33204 Gijón. Asturias. España. \\ ${ }^{2}$ Instituto de Productos Lácteos de Asturias. CSIC. Ctra. de Infiesto, s/n. 33300 \\ Villaviciosa. Asturias. España. \\ ${ }^{3}$ Instituto de la Grasa de Sevilla. CSIC. Avda. Padre García Tejero, 4. 41012 Sevilla. España.
}

\section{RESUMEN}

Estudio teórico de algunas propiedades termodinámicas de los ácidos grasos obtenidos como destilados en la desodorización de aceites comestibles

Actualmente se investiga la viabilidad de un cambio en la tecnología de la desodorización de aceites comestibles que afecta a la composición de los destilados que se obtienen en el proceso y al tratamiento de los mismos. El diseño de los equipos que conlleva este cambio de tecnología requiere el estudio de propiedades físicas de los destilados. Se han estudiado algunas propiedades termodinámicas de los ácidos palmítico, esteárico, oleico y linoleico, componentes mayoritarios resultantes del proceso de desodorización.

Las propiedades estudiadas han sido las correspondientes al punto crítico (temperatura, presión y volumen críticos), las curvas de presión de vapor en función de la temperatura y el calor latente de vaporización.

Se han recogido distintos métodos de estimación de las mencionadas propiedades y algunos datos experimentales, y se han comparado los resultados obtenidos.

PALABRAS-CLAVE: Ácidos grasos - Calor latente de vaporización - Curvas de presión de vapor - Propiedades termodinámicas (estimación) - Punto crítico.

\section{SUMMARY}

Theoretical study of some thermodynamic properties of the fatty acids obtained as distillates coming from the deodorization of vegetable oils

Nowadays the feasibility of a change of technology in the deodorization stage of edible vegetable oil refining is being investigated. The proposed technology involves a modification in the composition of the distillates coming from the deodorization stage and their further treatment. To design the apparatus which are required by the proposed technology it is necessary to study the physical properties of the distillates. The thermodynamic properties of the fatty acids: palmitic, stearic, oleic and linoleic, which are the principal components produced at the deodorization stage have been studied.

The properties that have been studied were those corresponding to the critical point (critical temperature, pressure and volume), the vapour pressure-temperature relation and the latent heat of vaporisation.

Different methods to estimate the above mentioned properties and some experimental published data have been compared.

KEY-WORDS: Critical point - Fatty acids - Latent vaporisation heat - Thermodynamic properties (estimation) - Vapour pressuretemperature relation.

\section{INTRODUCCIÓN}

Durante el proceso de desodorización se eliminan ciertas sustancias volátiles, algunas de las cuales dan al aceite olores y sabores desagradables. Estas sustancias son: ácidos grasos libres, algunos hidrocarburos, aldehídos y cetonas, peróxidos, pigmentos, etc. También se eliminan parcialmente, sin que sea deseable, algunos componentes que confieren al aceite parte de sus cualidades, como son esteroles y tocoferoles.

La desodorización se efectúa sometiendo al aceite neutro a un arrastre con vapor de agua saturado seco, bajo condiciones de presión y temperatura que dependen del tipo de aceite y de la procedencia de las semillas. La presión de desodorización suele estar comprendida entre 3 y $6 \mathrm{~mm} \mathrm{Hg}$ y se consigue utilizando termocompresores y eyectores con chorro de vapor. Los destilados de la desodorización, inicialmente en fase gas, se recuperan utilizando condensadores barométricos de contacto directo.

Actualmente se estudian cambios en la tecnología anteriormente descrita que afectan al modo de arrastre de los destilados de la desodorización, a la recuperación de los mismos y al gas utilizado en la desodorización. Estas modificaciones repercuten esencialmente en el consumo de agua, que se disminuye, y en una mayor recuperación y facilidad de separación de los destilados recogidos.

Respecto al arrastre de los destilados de desodorización, se sustituyen los eyectores por bombas de vacío. En cuanto a la recuperación de destilados, se sustituye la condensación de vapores en condensadores barométricos por la congelación de los mismos en congeladores de carcasa y tubos. La utilización de esta tecnología es posible utilizando como gas de arrastre vapor de agua saturado o nitrógeno. La utilización del nitrógeno como gas de arrastre ha sido investigada recientemente por Huesa et al. (1990), Graciani et al. (1994), Ruiz et al. (1996) y Dobarganes et al. (1996). 
En los trabajos previos sobre la tecnología propuesta se destaca su viabilidad en cuanto a rendimiento en la recuperación de destilados y a la calidad de los mismos. No obstante, es preciso abordar el diseño de los congeladores y del sistema de arrastre, a fin de garantizar unas condiciones óptimas en el proceso de desodorización de aquellas plantas de tratamiento de aceite que vayan a utilizar esta tecnología. Para proceder al diseño es necesario conocer previamente la composición de los destilados y, posteriormente, las propiedades físicas de las sustancias que forman los mismos y que afectan a la transferencia de calor y masa.

En el estudio de los componentes mayoritarios presentes en los destilados realizado por Ruiz et al. (1995), utilizando aceites de oliva, soja y girasol, se pone de manifiesto la importante presencia de los ácidos grasos palmítico (16:0), esteárico (18:0), oleiCo (18:1) y linoleico (18:2).

El objetivo del presente trabajo es la realización de un estudio bibliográfico para conocer algunas de las principales propiedades termodinámicas de los ácidos grasos. El estudio se ha centrado en los ácidos palmítico (16:0), esteárico (18:0), oleico (18:1) y linoleico (18:2). Las propiedades termodinámicas objeto de estudio son: las propiedades críticas (temperatura, presión y volumen críticos), la relación de presión de vapor - temperatura y el calor latente de vaporización en función de la presión de trabajo.

\section{PROPIEDADES CRÍTICAS}

Las propiedades críticas se usan en muchas técnicas de estimación de otras propiedades a través del concepto de estados correspondientes. Como los ácidos grasos se degradan térmicamente, las propiedades en el punto crítico deben ser estimadas.

\section{Temperatura crítica}

Se ha estimado la temperatura crítica de estos compuestos utilizando tres métodos:

- El propuesto por Lydersen, citado por Reid y Sherwood (1968), que relaciona la temperatura crítica con la temperatura normal de ebullición y la estructura molecular del compuesto en cuestión.

- El método propuesto por Forman y Thodos, citado por Reid y Sherwood (1968), que relaciona las propiedades del punto crítico con los parámetros de la ecuación de Van der Waals (fuertemente correlacionados con la estructura molecular).

- El método de Ambrose, citado por Reid et al. (1987), también basado en la estructura molecular del compuesto. Es más reciente que los anteriores y el recomendado cuando se conoce la temperatura de ebullición normal.

Como ya se ha mencionado, los métodos de Lydersen y de Ambrose precisan del conocimiento de la temperatura de ebullición normal. Para la determinación de la temperatura de ebullición normal de los ácidos palmítico, esteárico y oleico, se ha tomado la que se obtiene a partir de la relación presión de vapor - temperatura propuesta por Schlessinger, citado en CRC Handbook of Chemistry and Physics (1972-1973).

Para el ácido linoleico no se ha encontrado ninguna relacion presión de vapor - temperatura, disponiéndose solamente de pares de valores a presiones muy diferentes de la atmosférica. Por ello, para estimar la temperatura de ebullición normal, se ha teniendo en cuenta que los ácidos grasos insaturados presentan puntos de ebullición inferiores a los saturados correspondientes con el mismo número de átomos de carbono. (Martinenghi, 1950), y que los valores discretos disponibles se sitúan entre los correspondientes a los ácidos palmítico y oleico. Se ha tomado como temperatura de ebullición normal una situada entre las de los dos ácidos grasos anteriores.

\section{Presión crítica}

Se han probado otros tres métodos: el de Forman y Thodos, citado por Reid y Sherwood (1968); el de Ambrose, citado por Reid y Prausnitz (1987); y el de Riedel, citado por Reid y Sherwood (1968). En todos estos métodos la presión crítica es función de la estructura molecular y el peso molecular del compuesto.

\section{Volumen crítico}

Sólo se ha estimado según el método de Ambrose, citado por Reid y Prausnitz (1987), por ser más moderno y tener menor error porcentual medio frente a otros métodos.

Las propiedades críticas que se van a utilizar más adelante son las que proporciona el método de Ambrose, que es el más reciente y el que se recomienda en la bibliografía por estimar con un menor error. Los resultados de la aplicación de los diversos métodos se muestran en la tabla 1. 
Tabla I

Estimación de las propiedades críticas

\begin{tabular}{|c|c|c|c|c|c|c|c|}
\hline \multirow{2}{*}{ Ácido } & \multicolumn{3}{|c|}{$T_{c}(K)$} & \multicolumn{3}{|c|}{$P_{c} \times 10^{5}(\mathrm{~Pa})$} & \multirow{2}{*}{$\frac{V_{c}\left(m^{3} / \mathrm{kg}^{\prime}\right)}{\text { Ambrose }}$} \\
\hline & Lydersen & Forman-T. & Ambrose & Forman- $\mathrm{T}$. & Ambrose & Riedel & \\
\hline Palmítico & 777.05 & 779.05 & 785.15 & 15.76 & 14.68 & 15.12 & 0.003691 \\
\hline Esteárico & 790.55 & 795.65 & 795.35 & 13.96 & 13.26 & 13.63 & 0.003715 \\
\hline Oleico & 777.05 & 785.15 & 783.15 & 16.35 & 13.55 & 13.88 & 0.003670 \\
\hline Linoleico & 774.25 & 774.25 & 781.45 & & & & 0.003625 \\
\hline
\end{tabular}

\section{RELACIÓN DE PRESIÓN DE VAPOR - TEMPE- RATURA}

Se han utilizado relaciones de presión de vapor - temperatura procedentes de cuatro fuentes distintas:

- Los datos en forma de tabla propuestos por Pool y Ralston, citados por Formo et al. (1979), que relacionan la presión de vapor con la temperatura para los ácidos grasos saturados palmítico y esteáriço, para presiones de vapor desde $1 \mathrm{~mm} \mathrm{Hg}$ hasta $760 \mathrm{~mm} \mathrm{Hg}$.

- Los datos en forma de tabla propuestos por Jordan, citados por Gunstone et al. (1986), que relacionan presión de vapor - temperatura para los ácidos palmítico, esteárico y oleico, para presiones de vapor desde $1 \mathrm{~mm} \mathrm{Hg}$ hasta $760 \mathrm{~mm} \mathrm{Hg}$.

- La relación obtenida por Lederer, citado por Markley (1947), expresada por la ecuación 1:

$$
\ln P=-\frac{\lambda_{0}}{R T}+1.75 \ln T-\frac{\varepsilon T}{R}+C \ln 10
$$

donde $P$ es la presión de vapor $(\mathrm{mm} \mathrm{Hg}), T$ es la temperatura (K), $R$ es la constante de los gases ( $1.985 \mathrm{cal} / \mathrm{mol} \mathrm{K}), \lambda_{0}$ es el calor molar de vaporización a la temperatura del cero absoluto (cal $/ \mathrm{mol})$, $\varepsilon$ es el coeficiente de temperatura de la diferencia entre el calor específico en los estados líquido y gaseoso y $C$ es una constante química convencional.

La ecuación es válida para los ácidos grasos saturados, pero, como puede verse en la tabla II, también se incluyen las constantes correspondientes al ácido oleico, que es insaturado.

\section{Tabla II}

Valor de las constantes de la ecuación 1

\begin{tabular}{cccc}
\hline \multirow{3}{*}{ Constante } & \multicolumn{3}{c}{ Ácido } \\
\cline { 2 - 4 } & Palmítico & Esteárico & Oleico \\
\hline$\lambda_{0}$ & 26050 & 27280 & 26660 \\
$\varepsilon$ & 0.0326 & 0.0317 & 0.0304 \\
$C$ & 11.568 & 11.636 & 11.340 \\
\hline
\end{tabular}

- La expresión propuesta por Schlessinger, citado en CRC Handbook of Chemistry and Physics (1972-1973), que utiliza dos constantes específicas para cada compuesto $(A$ y $B)$ :

$$
\log P=-0.2185 \frac{A}{T}+B
$$

dônde $P$ es la presión de saturación $(\mathrm{mm} \mathrm{Hg})$, $A$ es el calor molar de vaporización (cal/mol) y $B$ es una constante específica para cada compuesto. En la tabla III se muestran los valores de $A$ y $B$ para los ácidos palmítico, esteárico y oleico, así como los intervalos de temperatura en los que son válidas las ecuaciones resultantes.

Las tablas IV, V, VI y VII recogen los resultados de la aplicación de las diferentes relaciones presión de vapor temperatura para los ácidos grasos palmítico, esteárico, oleico y linoleico, respectivamente. En la tabla del ácido oleico no figuran los resultados de la aplicación de la relación propuesta por Pool y Ralston, ya que el método se recomienda sólo para los ácidos grasos saturados.

Para el ácido linoleico se ha encontrado menos información que para los otros ácidos grasos, habiéndose obtenido tan sólo los dos pares de valores presión de vapor - temperatura que se muestran en la tabla VII, citados en The Merks Index (1989) y en CRC Handbook of Chemistry and Physics (1991-1992). La relación presión de vapor - temperatura suele utilizarse para estimar propiedades como el calor latente de vaporización, por lo que es conveniente disponer de una ecuación que exprese matemáticamente dicha relación también para este ácido. Se propone utilizar una expresión de la forma recomendada por Schlessinger y hacerla pasar por el punto de presión de vapor - temperatura $P=16 \mathrm{~mm} \mathrm{Hg}, \mathrm{T}=230^{\circ} \mathrm{C}$. Los valores de las constantes que resultan son: $A=19429,9$ y $B=9,65$.

Si se representa gráficamente la relación presión de vapor - temperatura a baja presión para los ácidos palmítico, esteárico y oleico, se obtienen curvas que no difieren sensiblemente para los diferentes métodos de estimación. Para los cálculos sucesivos se ha optado por la utilización de una expresión numérica para la relación presión de vapor - temperatura, prefiriéndose la recomendada por Schlessinger (ecuación 2), por ser la más actual. 
Tabla III

Valor de las constantes de la ecuación 2

\begin{tabular}{cccc}
\hline \multirow{2}{*}{ Constante } & \multicolumn{3}{c}{ Ácido } \\
\cline { 2 - 4 } & Palmítico & Esteárico & Oleico \\
\cline { 2 - 4 } & 17603.6 & 19306.6 & 20326.7 \\
$B\left(\mathrm{H}_{\mathrm{v}}, \mathrm{cal} / \mathrm{mol}\right)$ & 9.032695 & 9.457471 & 9.930301 \\
$B$ & $153.6-353.8$ & $173.7-370.0$ & $176.5-360.0$ \\
\hline (intervalo validez $\left.{ }^{\circ} \mathrm{C}\right)$ & & &
\end{tabular}

Tabla IV

Relación presión de vapor - temperatura para el ácido palmítico

\begin{tabular}{ccccc}
\hline \multirow{2}{*}{$\begin{array}{c}\text { Presión } \\
(\mathrm{mm} \mathrm{Hg})\end{array}$} & \multicolumn{4}{c}{ Temperatura $\left({ }^{\circ} \mathrm{C}\right)$} \\
\cline { 2 - 5 } & $\begin{array}{c}\text { Pool y } \\
\text { Ralston }\end{array}$ & Jordan & Lederer & Schlessinger \\
\hline 1 & 167.4 & 153.6 & 162.63 & 152.68 \\
2 & 179.0 & & 175.31 & 167.36 \\
4 & 192.2 & & 188.97 & 183.09 \\
5 & & 188.1 & 193.61 & 188.39 \\
8 & 206.1 & & 203.76 & 199.98 \\
10 & & 205.8 & 208.80 & 205.69 \\
15 & & & 218.32 & 216.42 \\
16 & 221.5 & & 219.89 & 218.18 \\
20 & & 223.8 & 225.40 & 224.33 \\
32 & 238.4 & & 237.58 & 237.82 \\
40 & & 244.4 & 243.66 & 244.49 \\
60 & & 256.0 & 255.25 & 257.05 \\
64 & 257.1 & & 257.16 & 259.11 \\
100 & & 271.5 & 270.97 & 273.78 \\
128 & 278.7 & & 279.08 & 282.25 \\
200 & & 298.7 & 294.71 & 298.24 \\
256 & 303.6 & & 303.97 & 307.48 \\
400 & & 329.0 & 321.98 & 324.98 \\
512 & 332.6 & & 332.77 & 335.13 \\
760 & 351.5 & 353.8 & 351.50 & 352.09 \\
\hline & & & & \\
\hline
\end{tabular}

Tabla V

Relación presión de vapor - temperatura para el ácido esteárico

\begin{tabular}{|c|c|c|c|c|}
\hline \multirow{2}{*}{$\begin{array}{l}\text { Presión } \\
(\mathrm{mm} \mathrm{Hg})\end{array}$} & \multicolumn{4}{|c|}{ Temperatura $\left({ }^{\circ} \mathrm{C}\right)$} \\
\hline & $\begin{array}{l}\text { Pool y } \\
\text { Ralston }\end{array}$ & Jordan & Lederer & Schlessinger \\
\hline 1 & 183.6 & 173.7 & 181.24 & 172.90 \\
\hline 2 & 195.9 & & 194.45 & 187.56 \\
\hline 4 & 209.2 & & 208.68 & 203.22 \\
\hline 5 & & 209.0 & 213.50 & 208.50 \\
\hline 8 & 224.1 & & 224.10 & 219.99 \\
\hline 10 & & 225.0 & 229.34 & 225.64 \\
\hline 15 & & & 239.27 & 236.24 \\
\hline 16 & 240.0 & & 240.90 & 237.97 \\
\hline 20 & & 243.4 & 246.64 & 244.05 \\
\hline 32 & 257.1 & & 259.34 & 257.32 \\
\hline 40 & & 263.3 & 265.68 & 263.87 \\
\hline 60 & & 275.5 & 277.77 & 276.18 \\
\hline 64 & 276.8 & & 279.76 & 278.19 \\
\hline 100 & & 291.0 & 294.18 & 292.52 \\
\hline 128 & 299.7 & & 302.64 & 300.77 \\
\hline 200 & & 316.5 & 318.97 & 316.32 \\
\hline 256 & 324.8 & & 328.64 & 325.28 \\
\hline 400 & & 343.0 & 347.47 & 342.20 \\
\hline 512 & 355.2 & & 358.76 & 351.98 \\
\hline 760 & 376.1 & 370.0 & 378.38 & 368.28 \\
\hline
\end{tabular}


Tabla VI

Relación presión de vapor - temperatura para el ácido oleico

\begin{tabular}{|c|c|c|c|}
\hline \multirow{2}{*}{$\begin{array}{l}\text { Presión } \\
(\mathrm{mm} \mathrm{Hg})\end{array}$} & \multicolumn{3}{|c|}{ Temperatura $\left({ }^{\circ} \mathrm{C}\right)$} \\
\hline & Jordan & Lederer & Schlessinger \\
\hline 1 & 176.5 & 175.69 & 174.11 \\
\hline 1.2 & & 179.00 & 177.70 \\
\hline 2 & & 188.67 & 188.09 \\
\hline 4 & & 202.67 & 202.97 \\
\hline 5 & 208.5 & 207.41 & 207.97 \\
\hline 8 & & 217.80 & 218.85 \\
\hline 10 & 223.0 & 222.94 & 224.19 \\
\hline 15 & & 232.67 & 234.19 \\
\hline 15.2 & & 232.99 & 234.53 \\
\hline 16 & & 234.26 & 235.82 \\
\hline 20 & 240.0 & 239.88 & 241.54 \\
\hline 29.5 & & 250.00 & 251.81 \\
\hline 30.0 & & 250.54 & 252.26 \\
\hline 32 & & 252.29 & 254.01 \\
\hline 40 & 257.2 & 258.47 & 260.14 \\
\hline 49 & & 264.40 & 265.85 \\
\hline 50 & & 264.87 & 266.42 \\
\hline 60 & 269.8 & 270.25 & 271.76 \\
\hline 64 & & 272.19 & 273.54 \\
\hline 100 & 286.0 & 286.19 & 286.90 \\
\hline 128 & & 294.39 & 294.58 \\
\hline 200 & 309.8 & 310.16 & 309.00 \\
\hline 256 & & 319.47 & 317.30 \\
\hline 400 & 334.7 & 337.54 & 332.91 \\
\hline 512 & & 348.31 & 341.91 \\
\hline 760 & 360.0 & 366.92 & 356.88 \\
\hline
\end{tabular}

Tabla VII

Relación presión de vapor - temperatura para el ácido linoleico

\begin{tabular}{ccc}
\hline \multirow{2}{*}{$\begin{array}{c}\text { Presión } \\
(\mathrm{mm} \mathrm{Hg})\end{array}$} & \multicolumn{2}{c}{ Temperatura $\left({ }^{\circ} \mathrm{C}\right)$} \\
\cline { 2 - 3 } & Merks Index Ed. 89 & $\begin{array}{c}\text { CRC Handbook } \\
\text { Ed. 91-92 }\end{array}$ \\
\hline 1.4 & 202 & \\
16 & 230 & $229-230$ \\
\hline
\end{tabular}

\section{CALOR LATENTE DE VAPORIZACIÓN}

Para obtener la relación calor latente de vaporización - temperatura, Markley (1947) propone la utilización de la ecuación de Clapeyron combinada con la relación presión de vapor - temperatura propuesta por Lederer. Markley también sugiere utilizar para todos los ácidos grasos una presión crítica entre 30 y $40 \mathrm{~atm}$. Estos valores no concuerdan con los estimados en el presente trabajo, ni con los estimados por otros autores. Esta cuestión, en condiciones de baja presión como las que se dan en la desodorización (4-6 mm $\mathrm{Hg}$ ), tiene una importancia secundaria, al tender a cero la relación $P / P_{c}$. En este trabajo se ha considerado una presión crítica para los ácidos grasos de $35 \mathrm{~atm}$. El resultado que se obtiene al tener en cuenta la recomendación de Markley se expresa en la ecuación 3:

$$
\lambda=\left(\lambda_{0}+1.75 R T-\varepsilon T^{2}\right)\left(1-\frac{P}{P_{c}}\right)
$$

donde $\lambda$ es el calor molar de vaporización a la temperatura considerada (cal $/ \mathrm{mol}), T$ es la temperatura considerada $(\mathrm{K}), P$ es la presión de saturación a la temperatura considerada $(\mathrm{mm} \mathrm{Hg}), P_{c}$ es la presión crítica $(\mathrm{mm} \mathrm{Hg}), \lambda_{0}$ es el calor molar de vaporización a la temperatura del cero absoluto (cal $/ \mathrm{mol}), \varepsilon$ es el coeficiente de temperatura de la diferencia entre el calor específico en los estados líquido y gaseoso y $R$ es la constante de los gases $(1.985 \mathrm{cal} / \mathrm{mol} \mathrm{K})$.

Otro método es el propuesto por Watson, citado por Reid et al. (1987), que expresa el calor latente de vaporización en función de la temperatura reducida y de un dato conocido de temperatura reducida - calor latente de vaporización. La expresión de la correlación de Watson se recoge en la ecuación 4:

$$
H_{v}=H_{v 1}\left(\frac{1-T_{r}}{1-T_{r 1}}\right)^{n}
$$

donde $H_{v}$ es el calor latente de vaporización a la temperatura reducida $T_{r}, H_{v 1}$ es el calor latente de vaporización conocido a determinada temperatura reducida $T_{r 1}$ y $n$ es una constante de la ecuación (se recomienda $n=0.38$ ). 
En este trabajo se ha aplicado el método de Watson al ácido palmítico y al ácido esteárico, tomando como datos del calor latente de vaporización en función de la temperatura los propuestos por Cramer, citado por Markley (1947), que se reproducen en la tabla VIII. Para el ácido palmítico se ha tomado como conocido el valor del calor latente de vaporización para la temperatura de $244^{\circ} \mathrm{C}$.

No se dispone de mucha información sobre el calor latente de vaporización en función de la temperatura para los ácidos oleico y linoleico, por lo que se ha buscado un método de estimación. La ecuación de RiedelPlank-Miller, citado por Reid et al. (1987), y expresado por la ecuación 5, estima el calor latente de vaporización en el punto de ebullición normal, con un error aproximado de $1.52 \%$ para los compuestos orgánicos:

$$
H_{v b}=2.303 G R T_{c}\left(1-\frac{0.96}{P_{c} T_{b r}}\right)\left[1+T_{b r}{ }^{2}+k\left(1+2 T_{b r}\right)\right]
$$

donde

$$
\begin{gathered}
G=0.2471+0.1965 a \\
k=\frac{a}{2.303 G}-\left(1+T_{b r}\right) \\
a=\frac{T_{b r} \ln P_{c}}{1-T_{b r}}
\end{gathered}
$$

siendo $H_{v b}$ el calor latente de vaporización a la ten:peratura de ebullición normal (cal/mol); $R$, la constante de los gases $(1.985 \mathrm{cal} / \mathrm{mol} \mathrm{K}) ; T_{c}$, la temperatura crítica $(\mathrm{K})$; $T_{b r}$, la temperatura de ebullición normal (K); y $P_{c}$, la presión crítica (atm).

Tabla VIII

Relación calor latente de vaporización - temperatura, según Cramer

\begin{tabular}{ccc}
\hline \multirow{2}{*}{ Ácido } & $T$ & $H_{v}$ \\
& $\left({ }^{\circ} \mathrm{C}\right)$ & (cal/g) \\
\hline \multirow{2}{*}{ Palmítico } & 202 & 84 \\
& 244 & 76 \\
\hline Esteárico & 242 & 67 \\
\hline
\end{tabular}

Esta ecuación tiene como parámetros la presión, la temperatura y el volumen críticos, además de la temperatura de ebullición normal. Teniendo en cuenta las temperaturas normales de ebullición utilizadas en la estimación de la temperatura crítica, así como los valores de temperatura y presión críticas estimadas por el método de Ambrose, se obtiene el calor latente de vaporización estimado para todos los ácidos grasos, según se muestra en la tabla IX.

Los resultados del estudio comparativo de los métodos de Markley y Watson se muestran en las tablas $X, X I$ y XII. La tabla X presenta los resultados para el ácido oleico, que también son válidos para el linoleico. Para estos últimos ácidos, en la aplicación del método de Watson se ha estimado el calor latente de vaporización a la temperatura de ebullición normal mediante la ecuación de Riedel-Plank-Miller.

Las figuras 1,2 y 3 muestran gráficamente la comparación de resultados de la aplicación de los métodos a baja presión. La forma de las ecuaciones obtenidas mediante ambos métodos es prácticamente la misma. El método propuesto por Markley proporciona valores inferiores a los obtenidos por el método de Watson-Cramer para el ácido palmítico y para los ácidos oleico y linoleico. Sin embargo, para el ácido esteárico los valores estimados según el método propuesto por Markley son superiores a los obtenidos por el método de Watson-Cramer.

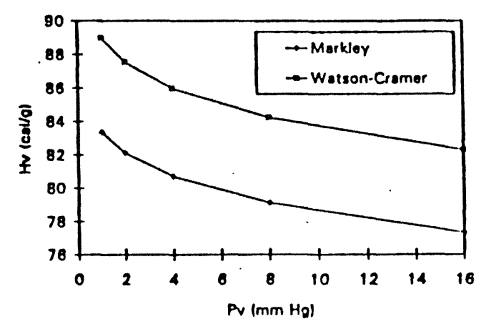

Figura 1

Calor latente de vaporización para el ácido palmítico

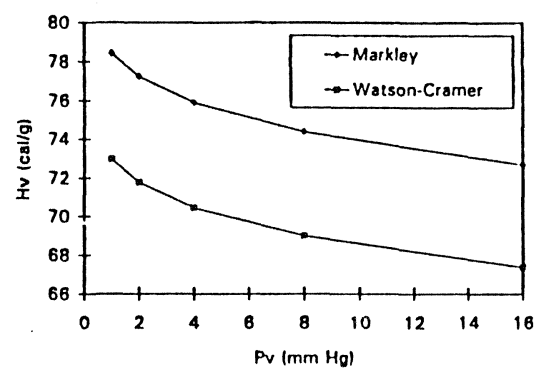

Figura 2

Calor latente de vaporización para el ácido esteárico

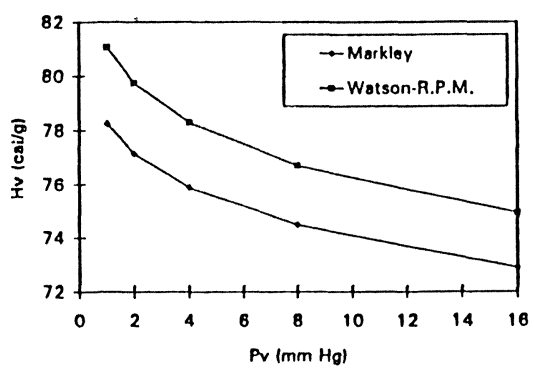

Figura 3

Calor latente de vaporización para el ácido oleico 
Tabla IX

Estimación del calor latente de vaporización, según Riedel-Plank-Miller

\begin{tabular}{ccccc}
\hline & \multicolumn{4}{c}{ Tipo de ácido } \\
\cline { 2 - 5 } Parámetro & Palmítico & Esteárico & Oleico & Linoleico \\
\hline$P M(\mathrm{~g} / \mathrm{mol})$ & 256.42 & 284.47 & 282.40 & 280.44 \\
$P_{c}(\mathrm{~atm})$ & 14.92 & 13.45 & 13.73 & 13.95 \\
$T_{c}(\mathrm{~K})$ & 777.88 & 790.55 & 777.08 & 775.20 \\
$T_{b r}(\mathrm{~K})$ & 625.20 & 641.40 & 630.03 & 627.00 \\
$H_{\text {bb }}(\mathrm{cal} / \mathrm{mol})$ & 16755.10 & 17031.48 & 16838.07 & 16722.12 \\
\hline
\end{tabular}

Tabla XI

Calor latente de vaporización para el ácido esteárico

Tabla X

Calor latente de vaporización para el ácido palmítico

\begin{tabular}{cccc}
\hline & Temperatura & \multicolumn{2}{c}{ Calor latente (cal/g) } \\
\cline { 3 - 4 } $\begin{array}{c}\text { Presión } \\
(\mathrm{mm} \mathrm{Hg})\end{array}$ & $\left({ }^{\circ} \mathrm{C}\right)$ & Markley & $\begin{array}{c}\text { Watson- } \\
\text { Cramer }\end{array}$ \\
\hline 1 & 181.24 & 78.43 & 72.96 \\
2 & 194.45 & 77.24 & 71.77 \\
4 & 208.68 & 75.90 & 70.45 \\
8 & 224.10 & 74.39 & 69.00 \\
16 & 240.90 & 72.69 & 67.38 \\
32 & 259.34 & 70.72 & 65.57 \\
64 & 279.76 & 68.42 & 63.52 \\
128 & 302.64 & 65.67 & 61.17 \\
256 & 328.64 & 62.69 & 58.45 \\
760 & 378.38 & 54.93 & 53.09 \\
\hline
\end{tabular}

Tabla XII

Calor latente de vaporización para el ácido oleico

\begin{tabular}{cccc}
\hline & & \multicolumn{2}{c}{ Calor latente (cal/g) } \\
\cline { 3 - 4 } $\begin{array}{c}\text { Presión } \\
(\mathrm{mm} \mathrm{Hg})\end{array}$ & $\begin{array}{c}\text { Temperatura } \\
\left({ }^{\circ} \mathrm{C}\right)\end{array}$ & Markley & $\begin{array}{c}\text { Watson- } \\
\text { Cramer }\end{array}$ \\
\hline 1 & 162.63 & 83.85 & 88.97 \\
2 & 175.31 & 82.09 & 87.55 \\
4 & 188.97 & 80.69 & 85.97 \\
8 & 203.76 & 79.11 & 84.23 \\
16 & 219.89 & 77.32 & 82.28 \\
32 & 237.58 & 75.26 & 80.09 \\
64 & 257.16 & 72.85 & 77.60 \\
128 & 279.08 & 69.96 & 74.74 \\
\hline 256 & 303.97 & 66.42 & 71.40 \\
760 & 351.50 & 58.72 & 64.78 \\
\hline
\end{tabular}

\begin{tabular}{cccc}
\hline \multirow{2}{*}{$\begin{array}{c}\text { Presión } \\
(\mathrm{mm} \mathrm{Hg})\end{array}$} & \multirow{2}{*}{$\begin{array}{c}\text { Temperatura } \\
\left({ }^{\circ} \mathrm{C}\right)\end{array}$} & \multicolumn{2}{c}{ Calor latente (cal/g) } \\
\cline { 3 - 4 } & 175.69 & Markley & Watson-RPM \\
\hline 1 & 188.67 & 77.12 & 81.06 \\
2 & 202.67 & 75.87 & 78.74 \\
4 & 217.80 & 74.48 & 76.69 \\
8 & 234.26 & 72.89 & 74.93 \\
16 & 252.29 & 71.06 & 72.96 \\
32 & 272.19 & 68.93 & 70.73 \\
64 & 294.39 & 66.39 & 68.21 \\
128 & 319.47 & 63.27 & 65.30 \\
256 & 366.92 & 56.51 & 59.65 \\
760 & & & \\
\hline
\end{tabular}




\section{CONCLUSIONES}

Se ha realizado un estudio de algunas propiedades termodinámicas de los ácidos grasos mayoritarios de los destilados de desodorización, y entre ellos, se han escogido para el estudio el palmítico, esteárico, oleico y linoleico.

La estimación de propiedades críticas por diversos métodos conduce a valores muy parecidos, y se propone el empleo del método de Ambrose como el más conveniente, por ser el correspondiente a una fuente más actual y basarse, además, en el dato experimental de la temperatura normal de ebullición, y no sólo en la estructura molecular del compuesto en cuestión.

Para las relaciones de presión de vapor - temperatura, se propone la utilización de la ecuación de Schlessinger, por ser la más actual, además de sencilla y de no presentar divergencias importantes con los valores presentados a través de tablas. Además, permite usar una expresión única para todos los ácidos, sin más que cambiar las constantes correspondientes.

En el caso del calor latente de vaporización, también se han obtenido valores similares en todas las fuentes consultadas, y se recomienda utilizar la expresión de Watson, por ser sencilla y permitir usar una expresión única para todos los ácidos grasos, sin más que cambiar el dato de partida del calor latente a una determinada temperatura para cada compuesto.

\section{AGRADECIMIENTOS}

Los autores hacen constar su agradecimiento a:

- La Comisión Interministerial de Ciencia y Tecnología, por las ayudas recibidas para la realización de este trabajo: ALI 91 0720, PTR 93 0061, ALI 940716 y ALI 950517.

- Las empresas Carburos Metálicos, S.A. y Busch Ibérica, S.A., por la colaboración y la tecnología prestada.

- Las empresas Aceprosa y Aceites Toledo, por los aceites suministrados.
- La American Soybean Association.

- Cuantos compañeros han hecho posible este trabajo.

\section{REFERENCIAS}

"CRC Handbook of Chemistry and Physics».- Ed. 53 (1972-1973).

"CRC Handbook of Chemistry and Physics".- Ed. 72 (1991-1992). Chemical Rubber Co.

Dobarganes García, $M^{\mathrm{a}}$ C.; Pérez Camino, $\mathrm{M}^{\mathrm{a}} \mathrm{C}$.; Márquez Ruiz, G.; Ruiz Méndez, $M^{\mathrm{a}} \mathrm{V}$. - «Comparative Performance of Steam and Nitrogen as stripping Gas in Physical Refining of Edible Oils".- J Journal of Am. Oil Chem. Soc. 72 (1996).

Formo; Jungermann; Norris; Sonntag. - «Bailey's Industrial Oil and Fat Products».- Vol. 1, 4th Ed., Edited by Daniel Swern, 1979.

Graciani Constante, E.; Bada Gancedo, J. C.; Rodríguez Berbel F. C.; Ruiz Méndez, Mª V.- - «Physical refining of oils using nitrogen as stripping gas. Process optimization».Grasas y Aceites 45 (1994), 132-146.

Gunstone, F. D.; Harwood, J. L.; Padley, F. B.— «The Lipid Handbook».- Chapman and Hall Ltd., 1986.

Huesa Lope, J.; Dobarganes García, M ${ }^{\mathrm{a}}$ C.- Patente $\mathrm{N}^{\mathrm{O}}$ 2013206 (1990). - Chem. Abstr. 113 (1990) 174464 w.

Markley K. S. - «Fatty Acids».- Interscience Publisher, Inc., New York, 1947.

Martinenghi, B.- «Aceites, Grasas y Derivados».- Traducción de 1950 de la $2^{\text {a }}$ edición en italiano.

Reid R. C.; Sherwood T. K.- «Propiedades de los Gases y Líquidos. Su Estimación y Correlación».- Traducción de la $2^{\mathbf{a}}$ edición de la versión inglesa editada por McGraw-Hill, UTHEA, 1968.

Reid R. C.; Prausnitz J. M.; Poling B. E.- «The Properties of Gases \& Liquids".- 4th Ed., McGraw-Hill, New York, 1987.

Ruiz Méndez, Mª V.; Márquez Ruiz; Dobarganes García, M ${ }^{a}$ C. - «Determinación Cuantitativa de Componentes Mayoritarios Presentes en los Destilados Obtenidos en la Desodorización de Aceites y Grasas".Grasas y Aceites 46 (1995), 21-15.

Ruiz Méndez, $M^{\mathrm{a}}$ V.; Garrido Fernández A.; Rodríguez Berbel F. C.; Graciani Constante, E.- «Relationships among the Variables Involved in the Physical Refining of Olive Oil Using Nitrogen as Stripping Gas».-Fat/Lipid 98 (1996), 121-125.

«The Merks Index».- 11th Ed., 1989. 